hep-th/0101010

SU-ITP-0038

UCSD-PTH-00-35

December 2000

\title{
Comments on Unstable Branes
}

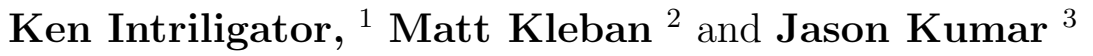 \\ ${ }^{1,3}$ Department of Physics, University of California, San Diego \\ La Jolla, CA 92093-0354 USA \\ 2 Department of Physics, Stanford University \\ Stanford, CA 94305 USA
}

\begin{abstract}
We argue that type II string theories contain unstable NS4 branes, which descend from a conjectured unstable M4 brane of M-theory. Assuming that an M2 brane can arise in M5 brane/anti-brane annihilation, the unstable M4 brane, and also an unstable M3 brane, must exist as sphalerons. We compare the tensions of the unstable NS4 branes, M4 brane, and related type II unstable D-branes, and present 11d supergravity solutions for unstable Mp branes for all $p$. We study the $Z_{2}$ gauge symmetry on the worldvolume of unstable branes, and argue that it can never be unbroken in the presence of lower brane charge.
\end{abstract}

\footnotetext{
${ }^{1}$ email address:keni@ucsd.edu

${ }^{2}$ e-mail address:mkleban@itp.stanford.edu

${ }^{3}$ e-mail address:j1kumar@ucsd.edu
} 


\section{Introduction}

The study of unstable brane systems has been an important direction in string theory research, following the work of [1, 2]. In the context of type IIA(B) string theory, simple examples are a Dp- $\overline{\mathrm{Dp}}$ system and a single $\mathrm{D}(p-$ 1 ) brane, with $p$ even (odd). These systems can decay to the closed string vacuum or to a state with some stable branes of lower dimension, as reviewed in the next section.

In this work we consider aspects of unstable brane systems and the $S L(2, Z)$ duality of type IIB from the point of view of M theory. For the most part, the study of M-theory has focused on the study of stable supersymmetric objects. However, there are compelling theoretical and phenomenological reasons for attempting to understand configurations in which supersymmetry is broken. Historically, the understanding of the structure of the atom depended greatly on the study of radioactive decay processes. It would be interesting to see if, in a similar way, the study of the decay of unstable branes gives any insight into the nature of the fundamental degrees of freedom of M-theory.

One might worry that it would be hopeless to follow non-BPS configurations from small to large string coupling. However, as discussed in [3], unstable branes can be regarded as sphalerons whose existence is protected by BPS charges. Perhaps, then, it is sensible to extrapolate to strong coupling. Other works which consider unstable branes in the context of S-duality and M-theory include [四, which considered general unstable Mp branes.

In IIB string theory we can S-dualize the two sides of $\mathrm{Dp}-\overline{\mathrm{Dp}} \rightarrow \mathrm{D}(p-2)$. We obtain, for example, NS5- $\overline{\mathrm{NS} 5} \rightarrow$ D3 (more generally, we can consider $(p, q)$ 5-branes). Likewise, we can $\mathrm{S}$-dualize the unstable $\mathrm{D}(2 \mathrm{n})$-branes to unstable NS(2n)-branes or, more generally, unstable $(p, q)$ 2n-branes. We'll focus on the unstable NS4 brane. The unstable NS4 can decay to the vacuum or, if the tachyon field condenses in a kink configuration, to a D3 brane.

By compactifying the IIB theory and T-dualizing to IIA, we argue that the unstable NS4 of IIB implies the existence of an unstable NS4 brane of IIA. The unstable IIA NS4 brane can decay to the vacuum or, if there are vortices, to D2 branes. In this way, the single unstable IIA NS4 is similar to the unstable D4- $\overline{\mathrm{D} 4}$ system, though the vortex is associated with the center of mass $U(1)$. This suggests that there can be an intermediate unstable NS3, in analogy with the IIA unstable D3, which can also decay to the D2 brane via a kink. 
We also consider uplifting unstable IIA brane systems to M-theory. The unstable IIA NS4 brane should correspond to an unstable M4 brane. The unstable M4 can decay to the vacuum or, if there are vortices in the worldvolume gauge field, to M2 branes. As with the NS3 brane mentioned above, we should also expect an intermediate unstable M3, which can decay to a M2 brane via a kink. As discussed in [5], the M2 can arise from M5- $\overline{\mathrm{M} 5}$ annihilation with a unit of $\mathrm{H}_{3}$ flux. The unstable M4 and M3 can then be regarded as sphalerons, along the lines of [3], whose existence is protected by the same non-trivial configuration space topology as leads to the M2 in M5- $\overline{\mathrm{M} 5}$ annihilation.

The conjectured unstable NS4 branes of IIA and IIB string theory are discussed in sect.3. The unstable M4 and M3 branes are discussed in sect. 4. In sect. 5 the connection between the unstable M-branes and IIA branes is discussed in more detail. The unstable IIA D3 brane, for example, is interpreted as an unstable M4 brane wrapped along the dilaton direction. The tensions of these unstable branes are compared and the action of IIB S-duality is further discussed. Section 6 contains 11d SUGRA solutions associated with the unstable M-branes, along the lines of [6].

Section 7 is devoted to a somewhat separate topic: the $Z_{2}$ symmetry of unstable type II branes, under which the real tachyon transforms as $T \rightarrow-T$. This $Z_{2}$ is actually a gauge symmetry (a fact which played an important role in [11]), which is Higgsed in the vacuum by the nonzero $T$ expectation value. We discuss some issues associated with the $Z_{2}$ gauge symmetry, such as the fact that the worldvolume coupling $\int d T \wedge C$ would not be $Z_{2}$ gauge invariant unless the RR field $C$ is also $Z_{2}$ charged, $C \rightarrow-C$ (this was also suggested to us by A. Sen []]). A nonzero $C$ expectation value then also spontaneously breaks the $Z_{2}$ gauge symmetry.

\section{Review}

As is well known, a coincident Dp- $\overline{\mathrm{Dp}}$ brane system has a worldvolume $U(1) \times$ $U(1)$ gauge theory, which contains a complex scalar field $T$ charged under the relative $U(1)$. The worldvolume theory is an Abelian Higgs model, with a "Mexican hat" potential for $T$. The potential has a local maximum at $T=0$ and a global minimum at $|T|=T_{0}$, with $V\left(|T|=T_{0}\right)+2 T_{p}=0$, so that the energy of the condensed "tachyon" $T$ cancels the tension of the Dp- $\overline{\mathrm{Dp}}$ system. Having $T$ relax to some fixed $\langle T\rangle$, with $|\langle T\rangle|=T_{0}$, is interpreted as 
annihilation of the brane-antibrane system to the closed string vacuum [2].

More generally, a Dp- $\overline{\mathrm{Dp}}$ pair, with $n$ units of magnetic flux, carries the charge of $n \mathrm{D}(p-2)$ branes, which is the final product of the brane annihilation [1]. Upon adding the $n$ units of magnetic flux to the worldvolume Abelian Higgs theory, the finite energy solution is a codimension 2 vortex, with $T$ winding precisely $n$ times around its minimum:

$$
n=\frac{1}{2 \pi} \oint A=\frac{1}{2 \pi i} \oint d(\ln T),
$$

where the integral is over a large circle surrounding the vortex. This correlation between the number of units of magnetic flux and the winding number of $T$ follows from the fact that the vortex configuration only has finite energy if the covariant derivative of $T$ vanishes far from the vortex, $\left(\partial_{\mu}+i A_{\mu}\right) T \rightarrow 0$ as $r \rightarrow \infty$. So if, for example, there is $n=1$ unit of $\mathrm{D}(p-2)$ brane charge, there is a vortex configuration where $T$ winds precisely once; likewise, if there is no $\mathrm{D}(p-2)$ brane charge, $n=0$, there cannot be a finite energy vortex configuration with winding $T$ : only the trivial vacuum is allowed. The vortex carries the Ramond-Ramond charge of $n \mathrm{D}(p-2)$ branes thanks to a worldvolume coupling $\sim \int F \wedge C$.

Sen further argues that one can consider an intermediate unstable $\mathrm{D}(p-1)$ brane in the decay of $\mathrm{Dp}-\overline{\mathrm{Dp}}$ to the vacuum or a $\mathrm{D}(p-2)$ brane. The intermediate unstable $\mathrm{D}(p-1)$ brane has a real tachyon $T$ and an even potential $V(T)=V(-T)$, with $T=0$ unstable, and stable minimum at some $|T|=T_{0} \neq 0$. The vacuum where $|T|=T_{0}$ is interpreted as the closed string vacuum, with $V\left(T_{0}\right)$ canceling off the $\mathrm{D}(p-1)$ brane tension: $V\left(T_{0}\right)+T_{p-1}=0$. The tachyon can also condense to a kink configuration, with $T \rightarrow+T_{0}$ for $x_{t} \rightarrow+\infty$ and $T \rightarrow-T_{0}$ for $x_{t} \rightarrow-\infty$ (where $x_{t}$ is the transverse coordinate to the codimension 1 domain wall). The domain wall is interpreted as the $\mathrm{D}(p-2)$ brane to which the unstable $\mathrm{D}(p-1)$ brane can decay. In the type II context, there must be a world-volume coupling [2]

$$
\int d T \wedge C_{p-2},
$$

in order for the $T$ kink configuration to carry the Ramond-Ramond charge of a $\mathrm{D}(p-2)$ brane. 


\section{Unstable NS-4-Branes}

We start by considering the S-dual of the D5- $\overline{\mathrm{D} 5} \rightarrow \mathrm{D} 3$ brane decay process in Type IIB string theory. Although it is notoriously difficult to follow non-supersymmetric states through S-duality, one nevertheless can easily understand both the beginning and end of the condensation path. By widely separating the D5- $\overline{\mathrm{D} 5}$, the system becomes meta-stable and S-dualizes to a NS5- $\overline{\text { NS5 }}$ brane system. The S-dual of the final product D3 brane is again a D3 brane. So a coincident NS5- $\overline{\mathrm{NS} 5}$-brane pair, with magnetic flux turned on, will decay to D3-branes. In analogy with the unstable D4, we claim that there exists an unstable NS4, which can be regarded as an intermediate state in the NS5- $\overline{\mathrm{NS} 5} \rightarrow D 3$ decay process.

The IIB NS5- $\overline{\mathrm{NS}} 5$ worldvolume theory is a $U(1) \times U(1)$ gauge theory, with complex tachyon charged under the relative $U(1)$, in complete analogy with the D5- $\overline{\mathrm{D} 5}$. There should be an unstable local maximum of the potential at $T=0$ and global minimum at $|T|=T_{0}$, with $2 T_{N S 5}+V\left(T_{0}\right)=0$, canceling off the tension of the two NS5 branes. The branes can annihilate to the vacuum or, with $n$ units of magnetic flux in the relative $U(1)$, there is a codimension 2 vortex, with winding number $n$, which is interpreted as the $n$ D3 branes to which the NS5- $\overline{\mathrm{NS} 5}$ (with $n$ units of flux) decays.

In the NS5- $\overline{\mathrm{NS} 5} \rightarrow \mathrm{D} 3$ vortex configuration, the tachyon may happen to condense into two domains separated by an unstable domain wall. If the tachyon condenses to positive real values in one domain, and negative real values in the other, then it interpolates through $T=0$ in the domain wall. $T=0$ corresponds to the state where the tachyon has not condensed and is described by the NS5- $\overline{\mathrm{NS} 5}$, where D-strings can end. So, in analogy with the heuristic arguments for the unstable D4-brane [2], IIB string theory has an unstable $4+1$ object, on which D-strings can end; this is the IIB unstable NS4 brane. The NS5- $\overline{\text { NS5 }}$ brane system can decay to an unstable NS4-brane by the condensation of one real tachyon and the NS4, in turn, decays to a D3-brane by the kink condensation of another real tachyon.

We can now consider the T-dual of this system. Suppose that one direction, which is common to each brane in NS5- $\overline{\mathrm{NS} 5} \rightarrow \mathrm{NS} 4 \rightarrow \mathrm{D} 3$, is taken to be compact (there cannot be a vortex on a compact direction without an anti-vortex). T-dualizing along this circle gives a IIA system, with a coincident NS5- $\overline{\mathrm{NS} 4} \rightarrow \mathrm{NS} 4 \rightarrow \mathrm{D} 2$. The fact that the unstable IIB NS4 brane gets T-dualized to a IIA unstable NS4 brane can be seen by studying the D-strings which begin and end on it. Under T-duality along a longitudinal 
direction, they become D2-branes which begin and end on a $4+1$ dimensional hypersurface. In analogy with the IIA NS5-brane (on which membranes can also begin and end), we call this surface the IIA unstable NS4-brane. Membranes intersects the NS4 brane on a world-volume string, which couples to a world-volume two-form tensor gauge field. In $4+1$ dimensions, strings coupled to two-form gauge fields are electric-magnetically dual to particles coupled to ordinary gauge fields. We therefore conjecture that both the IIA and IIB NS4 branes have a world-volume $U(1)_{c} \times Z_{2}$ gauge theory, where the $U(1)_{c}$ is the "center of mass" $U(1)$ of the NS5- $\overline{N S 5}$ system. In the IIB case, the stable D3 brane comes from a $Z_{2}$ kink. In the IIA case, the stable D2 brane comes from a vortex in $U(1)_{c}$, which Higgses $U(1)_{c}$. The $U(1)_{c}$ charged tachyon here is a nonperturbative state, along the lines of [5, 8].

\section{Unstable M4 and M3-Branes}

We now consider lifting the type IIA decay NS5- $\overline{\mathrm{NS} 5} \rightarrow \mathrm{NS} 4 \rightarrow$ D2 to Mtheory. The NS5- $\overline{\mathrm{NS} 5}$ becomes a M5- $\overline{\mathrm{M} 5}$, at a point on the dilaton circle. Likewise, the D2 becomes an M2 at a point on the dilaton circle. We therefore expect that the intermediate unstable NS4 becomes an unstable M4 brane, again at the same point on the dilaton circle. We thus have an M5- $\overline{\mathrm{M} 5}$ brane pair, with an appropriate $H_{3}$ magnetic flux, decaying to an unstable M4 brane, which will further decay to an M2-brane. The direct decay of the M5- $\overline{\mathrm{M} 5}$-brane pair, with $\mathrm{H}_{3}$ flux, to a M2-brane was described in [5].

The M5- $\overline{\mathrm{M} 5}$-brane pair has a $U(1)_{1} \times U(1)_{2}$ tensor multiplet theory living

on its worldvolume, with tachyonic strings (which are charged under the relative $U(1)$ ) coming from M2 membranes stretched between the 5-branes. The M4 brane is then also a place where M2 membranes can end, so its worldvolume gauge theory naturally has two-form gauge fields, with charged strings. The expected gauge group is $U(1)_{c} \times Z_{2}$, which is analogous to that of type II unstable branes. As with the IIA NS4 brane, in 5d we can Hodge dualize the two-form gauge fields and electrically charged strings to ordinary gauge fields coupled to electrically charged particles. Thus the $U(1)_{c}$ in the unstable M4 worldvolume can be described as an ordinary gauge theory, with electrically charged particles. (The worldvolume $Z_{2}$ gauge symmetry is perhaps more complicated, since the Hodge-duality between tensors and vectors need not directly apply to discrete gauge symmetries, which do not have propagating gauge fields.) 
We expect that the M4 worldvolume gauge theory has a complex tachyon, which is electrically charged under the $U(1)_{c}$ gauge group. This is similar to the $U(1)_{c}$ charged non-perturbative tachyon of [5, 8], whose condensation was argued to confine $U(1)_{c}$. In the present context, a configuration with $U(1)_{c}$ magnetic flux can have a codimension two vortex, where this complex tachyon winds around its $S^{1}$ vacuum configuration space. This vortex is the M2 brane to which the M4 brane can decay.

As usual, we might expect that the M4 $\rightarrow$ M2 decay, via a vortex, can occur via an intermediate unstable state: an unstable M3 brane, which can decay to the M2 brane via a kink in its worldvolume real tachyon scalar. Since there is no stable M1 brane, the M3 brane worldvolume gauge theory must not admit stable codimension 2 vortices.

The M4 and M3 branes can be regarded as sphaleronst, whose existence is related to that stable M2 branes. Consider the scenario of [5], where a M5- $\overline{\mathrm{M} 5}$ with $\mathrm{H}_{3}$ flux can decay to a stable M2 brane. The M2 is some codimension 3 solution for the fields in the M5- $\overline{\mathrm{M} 5}$ worldvolume; its stability should be protected by non-trivial $\pi_{2}$ topology. We can now write the same solution, but with some of the three transverse coordinates to the M2 replaced with parameters. Replacing one coordinate with a parameter $\sigma$, which lives on a circle $\sigma \in[0,2 \pi]$, we get a 1 -parameter family of codimension 2 solutions. Taking the vacuum to be at $\sigma=0$, the M3 brane is the sphaleron at $\sigma=\pi$. The one negative mode, associated with sliding down the circle, is interpreted as the real tachyon of the M3 brane, which can lead to a M2 brane via a kink.

Similarly, we can replace two of the coordinates in the M2 brane solution with parameters living on an $S^{2}$, to obtain a family of codimension 1 configurations. Taking the configuration at the south pole of the $S^{2}$ to be the vacuum, the configuration at the north pole is our unstable M4 brane sphaleron. There are two real unstable modes, associated with sliding down the $S^{2}$, which are interpreted as the complex tachyon in the M4 brane worldvolume; a vortex in this tachyon leads to the M2 brane. The existence of the unstable M3 and M4 branes are thus guaranteed by the same non-trivial $\pi_{2}$ topology which ensures the stability of the BPS M2 brane configuration in the M5- $\overline{\mathrm{M} 5}$ annihilation process.

The existence of the M4 brane is also suggested by the work of [9] on

\footnotetext{
${ }^{4}$ See the recent discussion in [3] (in particular Figs. 3 and 4), and the references cited therein, for the relevant background on sphalerons.

${ }^{5}$ We thank O. Bergman for pointing this out to us.
} 
non-BPS states in the heterotic theory on $T^{4}$ and type IIA on K3. The dual of the $Z_{2}$ valued D-particle seen in the type I string was argued there to arise from a non-BPS 4-brane in IIA or M theory, which is wrapped on the $T^{4} / Z_{2}$.

In [10] it was further suggested that end-of-the-world M9- $\overline{\mathrm{M} 9}$ branes can annihilate to form M5 branes, which are associated with $\pi_{3}\left(E_{8}\right)=Z$. If so, along the lines of the discussion above, one should also expect unstable M8, M7, and M6 branes as sphalerons, whose existence is protected by the same $\pi_{8}\left(E_{8}\right)$ topology as gives the M5 brane.

\section{Type II Branes from Unstable M-branes}

Consider the decay M5- $\overline{\mathrm{M} 5} \rightarrow \mathrm{M} 4 \rightarrow \mathrm{M} 2$, but now with one direction common to each of the branes wrapped along the dilaton direction. The wrapped M5-brane is the IIA D4-brane, the wrapped M2 brane is the IIA fundamental string, and we identify the wrapped M4 brane as the IIA unstable D3-brane. So the IIA decay is D4- $\overline{\mathrm{D} 4} \rightarrow \mathrm{D} 3 \rightarrow \mathrm{F} 1$. The IIA unstable D3 has a worldvolume $U(1)_{c} \times Z_{2}$ gauge theory, and the fundamental string arises via a $U(1)_{c}$ vortex. The $U(1)_{c}$ charged tachyon which winds in this vortex must arise non-perturbatively, along the lines of [5, 8]. The corresponding $\mathrm{M} 4 \rightarrow$ M2 decay presumably also occurs via a vortex, with winding charged tachyon vev, in the dualized $U(1)_{c}$ M4 worldvolume gauge theory.

A more standard IIA decay is D4- $\overline{\mathrm{D}} 4 \rightarrow \mathrm{D} 3 \rightarrow \mathrm{D} 2$, with the D4- $\overline{\mathrm{D}} 4$ system having a unit of magnetic flux in the relative $U(1)_{r}$ and the $Z_{2}$ charged, perturbative, real tachyon in the D3 brane worldvolume (which is $U(1)_{c}$ neutral) having a kink. This decay also corresponds to the M-theory decay $\mathrm{M} 5-\overline{\mathrm{M} 5} \rightarrow \mathrm{M} 4 \rightarrow \mathrm{M} 2$, with the M5- $\overline{\mathrm{M} 5}$ and the M4 again wrapped on the dilaton circle, but now with the M2 brane at a point in the dilaton direction. Since D3 $\rightarrow$ D2 occurs via a kink in the D3 worldvolume $Z_{2}$ gauge theory, presumably its M-theory uplift to M4 $\rightarrow \mathrm{M} 2$ should also involve a kink in the M4 worldvolume $Z_{2}$ tensor (rather than vector, and thus perhaps mysterious) gauge theory. This kink is presumably what distinguishes this case from the above $\mathrm{M} 4 \rightarrow \mathrm{M} 2$ decay, corresponding to $\mathrm{D} 3 \rightarrow \mathrm{F} 1$.

We now consider the tensions of the unstable branes. At weak coupling, the tension of a stable type II Dp brane is $T_{p}=1 / g(2 \pi)^{p} l_{s}^{p+1}$, whereas the tension of an unstable type II Dp brane is $T_{p}=\sqrt{2} / g(2 \pi)^{p} l_{s}^{p+1}$ [2]; numerical factors will henceforth be dropped. IIA tensions are lifted to M-theory using $g \propto R / l_{s}$ and $g l_{s}^{3}=l_{11}^{3}$. 
Interpreting the IIA unstable D3 as an unstable M4 wrapped on the dilaton circle, one would naively expect its tension to be given by $T_{D 3}=$ $\int_{0}^{2 \pi R} T_{M 4}$, with $R$ the radius of the dilaton direction and $T_{M 4}$ the tension of the unwrapped, unstable $M 4$-brane. Using $T_{D 3} \propto 1 / g l_{s}^{4}$ for $g \ll 1$, this gives

$$
T_{M 4} \propto \frac{1}{l_{11}^{\frac{9}{2}} R^{\frac{1}{2}}} \quad \text { for } \quad R \ll l_{11}
$$

where the $R \ll l_{11}$ restriction is because the D3 tension is only known for $g \ll 1$. The fact that the above tension is $R$ dependent might seem troubling, but is perhaps not entirely surprising. If, for example, the unstable M4 were placed on a transverse circle, one might expect an interaction energy between the M4 and its images. Of course this would not happen with BPS states, but for the unstable branes there is no supersymmetry to impose a no-force condition. So the effective tension of the M4 could depend on the radius of the transverse circle. Similarly the tension of the wrapped M4 could be expected to be radius dependent, as given above.

Naively applying the tension formula (3) outside of its region of validity would suggest that $T_{M 4} \rightarrow 0$ for $R \rightarrow \infty$. Assuming that the M4 exists as an unstable state in uncompactified M-theory, we would expect that (3) is not exact for all $R$, with $T_{M 4} \sim 1 / l_{11}^{5}$ in the $R \rightarrow \infty$ limit.

Consider wrapping the IIA unstable D3 brane on a circle of radius $R_{2}$. In M-theory we expect this to correspond to the M4 brane wrapped on a torus, with sides of length $R_{1} \sim g l_{s}$ (the dilaton direction) and $R_{2}$. For $g \ll 1$, we know that the tension of the unwrapped D3 is $T_{D 3} \propto 1 / g l_{s}^{4}$. Wrapping the D3 on the circle of radius $R_{2} \gg l_{s}$ gives a $2+1$ dimensional object with tension $T_{2+1} \propto R_{2} T_{D 3} \propto R_{2} / g l_{s}^{4}$ which, in terms of M-theory, is

$$
T_{2+1} \propto \frac{R_{2}}{g l_{s}^{4}}=\frac{R_{2} R_{1}^{\frac{1}{2}}}{l_{11}^{\frac{9}{2}}} \quad \text { for } \quad R_{1} \ll l_{11} \ll R_{2} .
$$

The expected invariance under exchanging $R_{1}$ and $R_{2}$ must be restored by corrections to (4) which are subleading in the above $R_{1} \ll l_{11} \ll R_{2}$ limit. Upon T-dualizing, this wrapped IIA D3 brane becomes an unwrapped, unstable IIB D2 brane. The requirement of invariance under exchanging $R_{1}$ and $R_{2}$ is then S-duality in the IIB coupling $g_{b}=R_{1} / R_{2}$.

\footnotetext{
${ }^{6} \mathrm{By} T_{p+1}$, we shall mean the tension of the $\mathrm{p}+1$ dimensional object obtained by considering the brane on a length scale such that all other (compact) directions are small. This tension has dimensions of $[\text { energy }]^{p+1}$.
} 
We next consider the type II unstable NS4 branes. A guess for their tension at weak coupling is $T_{N S 4} \propto 1 / g^{2} l_{s}^{5}$. This is compatible with the Tduality between the wrapped IIA NS4 and wrapped IIB NS4, since $R_{a} / g_{a}^{2} l_{s}^{5}=$ $R_{b} / g_{b}^{2} l_{s}^{5}$ (using $g_{b}=g_{a} l_{s} / R_{a}$ and $R_{b}=l_{s}^{2} / R_{a}$ ).

Assuming the $R_{1} \leftrightarrow R_{2}$ invariance, we can use (3) to obtain the tension of the IIA NS4 wrapped on a small circle of radius $R_{2}$, with $R_{2} \ll l_{11} \ll R_{1}$ :

$$
T_{3+1_{N S 4-I I A}} \propto \frac{R_{2}^{\frac{1}{2}}}{l_{11}^{\frac{9}{2}}}=\frac{R_{2}^{\frac{1}{2}}}{g_{a}^{\frac{3}{2}} l_{s}^{\frac{9}{2}}} \quad \text { for } \quad R_{2} \ll l_{11} \ll R_{1} .
$$

The tension (5) is a prediction for strong IIA coupling, $g_{a} \sim\left(R_{1} / l_{11}\right)^{3 / 2} \gg 1$; it differs from the above weak coupling guess $R_{2} / g_{a}^{2} l_{s}^{5}$. Now T-dualize, to get the IIB NS4 brane, wrapped on a circle of radius $R_{b}=l_{s}^{2} / R_{2}$. In terms of $R_{b}$ and the IIB coupling $g_{b}=R_{1} / R_{2}$, the tension (5) of this wrapped object is

$$
T_{3+1_{N S 4-I I B}} \propto \frac{R_{b}}{g_{b}^{\frac{3}{2}} l_{s}^{5}} \quad \text { for } \quad g_{b} \gg 1, \quad R_{b} \text { large. }
$$

Unlike the (strong coupling, small wrapping radius) tension (5), the (strong coupling, large radius) tension (6) has standard dependence on the wrapping radius $R_{b}$. Thus $T_{3+1_{N S 4-I I B}}=\int_{0}^{2 \pi R_{b}} T_{N S 4-I I B}$ gives

$$
T_{N S 4-I I B} \propto \frac{1}{g_{b}^{\frac{3}{2}} l_{s}^{5}}=\frac{1}{g_{b}^{\frac{1}{4}} l_{10 p}^{5}} \quad \text { for } \quad g_{b} \gg 1, \quad R_{b} \text { large }
$$

a radius independent tension. $l_{10 p}$ is the IIB, $S L(2, Z)$ invariant, 10 d Planck length. The tension (7) could be computed another way: its S-dual is

$$
T_{s} \propto \frac{g_{b}^{\frac{1}{4}}}{l_{10 p}^{5}}=\frac{1}{g_{b} l_{s}^{5}} \quad \text { for } \quad g_{b} \ll 1, \quad R_{b} \text { large }
$$

which is just the tension of the IIB unstable D4-brane. As might have been anticipated, the unstable NS4-brane of IIB is the S-dual of the unstable D4-brane of IIB.

The reader might wonder if this compatibility of the $T_{D 4}$ and $T_{N S 4}$ with S-duality really had any right to work. The above wrapped IIA NS4 brane tension (5) is only the leading order term in an expansion in $R_{2} / l_{11}$ and $R_{2} / R_{1}$. On the other hand, consider starting with the IIB unstable D4, 
whose tension is only known to leading order in $g_{B}=R_{1} / R_{2}$ and $R_{b}$ large. Upon S-dualizing, this gives the IIB NS4 brane tension to leading order in $1 / g_{B}=R_{2} / R_{1}$ and $R_{b}$ large. Upon T-dualizing, this gives the IIA NS4 brane tension in the limit of $R_{2}$ small compared to $R_{1}$ and $l_{11}$, which agrees with the region where our analysis of the wrapped IIA NS4 is valid. The compared tension is just the leading term of a double expansion, in $R_{2} / l_{11}$ and $R_{2} / R_{1}$, for both the wrapped NS4 of IIA and NS4 of IIB. The higher order corrections presumably also match, so that the NS4 of IIA and IIB are T-dual, and the NS4 and D4 of IIB are S-dual, for all coupling and radii.

\section{Unstable branes in 11d SUGRA}

In this section we find non-BPS solutions of 11d SUGRA corresponding to uncharged Mp branes, for arbitrary p. The ansatz for the Mp brane metric, in isotropic coordinates, is

$$
d s^{2}=e^{2 A(r)}\left(-d t^{2}+d x_{a} d x^{a}\right)+e^{2 B(r)}\left(d r^{2}+r^{2} d \Omega_{9-p}^{2}\right),
$$

where $a$ runs from 1 to $p$, with the 4 -form $H_{4}=0$ (since there is no charge). Einstein's equations then imply $R_{\mu \nu}=R=0$, which amounts to a set of differential equations for the functions $A(r)$ and $B(r)$ :

$$
\begin{gathered}
A^{\prime \prime}+(p+1) A^{2}-(p-8) A^{\prime} B^{\prime}-(p-9) A^{\prime} / r=0, \\
(p-9) B^{\prime \prime}-(p+1)\left[A^{\prime \prime}+A^{\prime}\left(A^{\prime}-B^{\prime}\right)\right]+(p-9) B^{\prime} / r=0, \\
B^{\prime \prime}+(8-p) B^{\prime 2}+(p+1) A^{\prime}\left(B^{\prime}+1 / r\right)+(17-2 p) B^{\prime} / r=0 .
\end{gathered}
$$

The most general solution to these equations is

$$
e^{2 A(r)}=\left(\frac{f_{-}(r)}{f_{+}(r)}\right)^{C}, \quad e^{2 B(r)}=\left(\frac{f_{+}(r)}{f_{-}(r)}\right)^{\frac{C(p+1)}{(8-p)}}\left[f_{+}(r) f_{-}(r)\right]^{2 /(8-p)},
$$

where

$$
f_{ \pm}(r) \equiv 1 \pm\left(\frac{r_{0}}{r}\right)^{8-p}, \quad C \equiv \frac{2}{3} \sqrt{\frac{9-p}{p+1}},
$$

and $1 / r_{0}$ is the mass parameter. We can convert from the above isotropic coordinate $r$ to a Schwarzschild coordinate $\rho$ via $\rho=e^{B} r$. The $p=0$ case of the above general solution is then recognized as the $11 \mathrm{~d}$ Schwarzschild metric

$$
d s^{2}=-\left(1-\frac{4 r_{0}^{8}}{\rho^{8}}\right) d t^{2}+\left(1-\frac{4 r_{0}^{8}}{\rho^{8}}\right)^{-1} d \rho^{2}+\rho^{2} d \Omega_{9}^{2} .
$$


The above metric (9), (13) properly asymptotes to flat $11 \mathrm{~d}$ spacetime in the $r \rightarrow \infty$ limit (provided $p<8$ ). The tension $T_{p}$ of the Mp brane can be found by expanding in the $r \rightarrow \infty$ limit

$$
g_{t t} \rightarrow-1+\text { const. } \frac{l_{11}^{9} T_{p}}{r^{8-p}}+\ldots ;
$$

this gives

$$
T_{p} \propto \frac{r_{0}^{8-p}}{l_{11}^{9}} .
$$

Note also that the above solution only makes sense for $r \geq r_{0}$, and has a singularity at $r=r_{0}$. For $p=0$ this is merely a coordinate singularity associated with the Schwarzschild horizon. For $p>0, r=r_{0}$ is truly singular, as can be seen in the curvature invariant $R_{\text {Riemann }}=R^{\mu \nu \lambda \sigma} R_{\mu \nu \lambda \sigma}$.

As might have been expected from Birkhoff and no-hair theorems, the above Mp brane solutions contain only a single parameter, the mass $1 / r_{0}$. To see this, note that the first of the three differential equations can be used to solve for $B^{\prime}$ in terms of $A^{\prime}$ and $A^{\prime \prime}$. Integrating $A^{\prime}$ to $A$ and $B^{\prime}$ to $B$ introduces trivial constants, which are fixed by the boundary condition that the solution approach the Minkowski solution at large $r$. That leaves two second order equations for a single variable. The first can be solved, with two undetermined constants. The second differential equation fixes one of these, leaving $r_{0}$ as the only free parameter.

The above 11d SUGRA solutions are analogous to type II SUGRA unstable brane solutions found in [6]. However, the solution of [6] depended on a second parameter, in addition to the mass $1 / r_{0}$. An argument similar to the one above indicates that the 10D solution should indeed depend on an additional parameter, due to the fact that the dilaton field can vary independently of the metric. No-hair theorems fail in this case, due to the presence of a curvature singularity. The additional parameter was interpreted in [6] as corresponding to the expectation value of the tachyon field.

A possible reason for why the type II case differs from M-theory (in having a parameter corresponding to the tachyon vev) is that IIA unstable branes are long lived at weak coupling. This is because the IIA unstable branes are expected to have a decay time on order $l_{s}$ which, at weak coupling, is long lived compared to the natural SUGRA time scale $l_{10 p}=g^{1 / 4} l_{s}$. On the other hand, unstable Mp branes should be expected to decay on time scales on order $l_{11}$, and thus meta-stable SUGRA solutions including the extra tachyon parameter should not be expected. 
KK reducing the 11d SUGRA action leads to the IIA SUGRA action via

$$
G=\left[\begin{array}{cc}
\lambda^{-1 / 8} g & 0 \\
0 & \lambda
\end{array}\right]
$$

where $G$ is the metric in 11 dimensions, $g$ is the 10 dimensional metric, and $\lambda$ the dilaton. KK reducing the above unstable Mp brane solutions then leads to unstable brane solutions of IIA SUGRA, along the lines of [6]. The metric ansatz and requirement that there be an isometry along the reducing direction implies that one can only dimensionally reduce these solutions along the brane, i.e. reducing the Mp solution to a $\mathrm{D}(\mathrm{p}-1)$ solution. The solution of [6] is then indeed obtained 7 . Of course, the classical 11d SUGRA solutions should really only be trusted when the dilaton circle is very large. They can thus be regarded as the unstable brane solutions of IIA string theory at very strong string coupling. On the other hand, the solutions of [6] should really only be trusted for weak string coupling, when the dilaton circle is very small. So we should not, for example, expect the ADM tension $T_{p}$ of the compactified 11d SUGRA solution to accurately give the tension IIA unstable branes for all string coupling (only very large coupling).

\section{Unstable branes and $Z_{2}$ gauge symmetry}

The worldvolume gauge theory of unstable Dp branes (p odd for IIA or even for IIB) is $U(1)_{c} \times Z_{2}$, where $U(1)_{c}$ is the "center of mass" $U(1)$ and the $Z_{2}$ acts on the real tachyon (which is $U(1)_{c}$ neutral) as $T \rightarrow-T$. An argument given in [1] for why this $Z_{2}$ is a discrete gauge rather than global symmetry follows from the construction of type IIA (IIB) unstable Dp branes by a $(-1)^{F_{L}}$ orbifold of the $\mathrm{Dp}-\overline{\mathrm{Dp}}$ brane system of IIB (IIA): $U(1)_{c} \times Z_{2}$ is the subgroup of the $U(1)_{c} \times U(1)_{r}$ gauge symmetry of the $\mathrm{Dp}-\overline{\mathrm{Dp}}$ brane system which survives under $(-1)^{F_{L}}$. Because it's a gauge transformation, $T \rightarrow-T$ is not really a symmetry but, rather, a redundancy of these variables.

We can define covariant derivatives $D T$, which transform as $D T \rightarrow \epsilon(x) T$ under $T(x) \rightarrow \epsilon(x) T(x)$, with $\epsilon(x)= \pm 1 ; D T$ differs from $d T$ only by terms of delta function support, needed to cancel the $(d \epsilon) T$ term. Wilson loops are $Z_{2}$

\footnotetext{
${ }^{7}$ The Mp solution reduces to the $\mathrm{D}(\mathrm{p}-1)$ solution of [6], with their parameters set to $c_{2}=-1$ and $c_{1}=3 C \frac{q-4}{7-q}$, with $q=p-1$, and we need to choose the negative branch in defining their parameter $k=-\frac{3}{4} C q$. In the Euclidean version, as in [3] (see their fig. (2)), the $\mathrm{D}(\mathrm{p}-1)$ brane has periodic fermion boundary conditions around the Euclidean time $S^{1}$.
} 
group elements, which can only be non-trivial on a space with nontrivial $\pi_{1}$ (either via $S^{1}$ compactification or by circling a string defect). Finite energy requires $D T \rightarrow 0$ at infinity. As in the vortex case eqn.(1), we might expect that this correlates the kink number

$$
\frac{1}{2 T_{0}}\left(T\left(x_{t}=\infty\right)-T\left(x_{t}=-\infty\right)\right)=\frac{1}{2 T_{0}} \int d x_{t} d T
$$

with $Z_{2}$ flux localized on the codimension 1 domain wall. Then the absence or presence of $Z_{2}$ flux is what decides whether the unstable brane decays to vacuum or a codimension 1 stable brane.

\subsection{Is the $Z_{2}$ Symmetry Broken?}

We note that the $d T \wedge C$ term (eqn. 2) in the worldvolume of an unstable Dp brane would break the $Z_{2}$ gauge invariance unless both $T$ and $C$ transform, $T \rightarrow-T$ and $C \rightarrow-C$, under a gauge transformation. The fact that the background field $C$ is charged means that any non-zero $C$ expectation value spontaneously breaks the $Z_{2}$ gauge symmetry.

The tachyon kink and anti-kink are related by $T \rightarrow-T$, and thus apparently $Z_{2}$ gauge equivalent. Since the kink and anti-kink correspond to a $\mathrm{D}(p-1)$ brane or anti-brane, they should not be equivalent. This can be resolved by saying that the $Z_{2}$ gauge symmetry is broken by two effects: the $T$ expectation value in the kink configuration and also by the fact that $C$ is charged. Then the $\mathrm{D}(p-1)$ brane and anti-brane can be inequivalent and correspond to an alignment or misalignment of these two $Z_{2}$ breakings.

To get another perspective on the $Z_{2}$ breaking, consider a $\mathrm{D}-(p+1)$ brane/anti-brane pair with a unit of magnetic flux in the relative $U(1)$, i.e. a unit of $\mathrm{D}(p-1)$ brane charge. This system can decay to an unstable D-pbrane, also with one unit of $\mathrm{D}$ - $(p-1)$-brane charge. Thus the unstable brane must have a kink, and the kink number cannot change. This means that, if there is non-zero $\mathrm{D}(p-1)$ brane charge, the tachyon must be condensed at $\pm \infty$. Because $T$ is $Z_{2}$ charged and necessarily condensed, the gauge symmetry is necessarily broken. It is not surprising that tachyon condensation breaks the $Z_{2}$ gauge symmetry. But it is surprising that the unstable brane with non-zero RR charge cannot ever have an unbroken $Z_{2}$ gauge symmetry, because the tachyon can never be uncondensed.

The condensation of the tachyon living on an unstable brane is the worldvolume signature that the unstable brane has decayed to the vacuum [2]. 
The fact that the tachyon must be condensed at infinity then corresponds to the statement that one cannot meaningfully discuss an infinite unstable D-p-brane. If it has non-zero D- $(p-1)$-brane charge, then the tachyon kink must always be non-zero, which implies that the brane has already decayed to the vacuum, at least at $\pm \infty$. From the spacetime point of view this is because, over an arbitrarily small time $\delta t$, the probability that the unstable brane decays within an area $A$ can be made arbitrarily close to 1 simply by increasing $A$. By the time the unstable D2-brane has formed, it has already decayed to the vacuum in an infinite number of places. For an unstable Dbrane, the decay constant is expected to be on the order of the string scale, i.e. for $\delta t$ of order the string scale, the probability that the brane has begun to decay within a string scale volume is $\sim 1 / e$.

\subsection{Multiple D-branes From a Single Unstable Brane?}

If a potential has only two minima, then a kink cannot be followed by another kink; it can only be followed by an anti-kink. This suggests that an unstable Dp brane can decay to at most a single $\mathrm{D}(p-1)$ brane; to get multiple $\mathrm{D}(p-1)$ branes would require multiple unstable Dp branes ([12], [13]).

On the other hand, a single $\mathrm{D}(p+1)$ brane/anti-brane pair can decay to an arbitrary integer $n \mathrm{D}(p-1)$ branes when there are $n$ units of magnetic flux, and thus $n$ vortices. We can construct domain walls with any number of flux units, such that the tachyon field interpolates through zero at the wall and the flux is contained within the wall; this implies that an unstable Dp brane can decay to any number of $\mathrm{D}(p-1)$ branes. A possible resolution is that this domain wall is really a set of $n$ coincident unstable Dp branes, each of which decays to a single $\mathrm{D}(p-1)$ brane via a kink. But note that a single unstable Dp brane can carry arbitrary fundamental string charge, which just corresponds to the electric flux of the $U(1)$. For the case of the unstable D3-brane, these fundamental strings are wrapped M2-branes, while the $\mathrm{D}(p-1)$-branes are unwrapped M2-branes. So we'd then have to explain why an unstable D3-brane can carry arbitrary wrapped M2-brane charge $n$, but can only have unwrapped M2-brane charges of magnitude 1 or 0 . This difference can not be explained by claiming that the $n$ fundamental strings are merely a single M2 brane wound $n$ times around the dilaton circle: the $n$ fundamental strings can be separated with no energy cost, unlike a multiply wound M2 brane.

A possible resolution of this puzzle is to conjecture that the tachyon 
potential is actually periodic, with an infinite number of global minima (as in the sine-Gordon model). In that case, any number of kinks could appear successively, allowing the unstable brane to have any integer number of RR charge. This conjecture seems to conflict with recent results in boundary string field theory [14, which found a non-periodic tachyon potential. But perhaps the domain of the BSFT tachyon actually corresponds to only a finite segment of a full periodic tachyon potential.

\section{Acknowlegements}

We are grateful to O. Bergman, J. McGreevy, J. Preskill, A. Sen, S. Shenker, and L. Susskind for useful discussions. The work of K. I. and J. K. is supported by DOE-FG03-97ER40546. M.K. is supported by Stanford University.

\section{References}

[1] A. Sen, "Tachyon Condensation on the Brane Antibrane System," hepth/9805170, JHEP 9808, 012 (1998).

[2] For a review and references, see A. Sen, "Non-BPS States and Branes in String Theory," hep-th/9904207.

[3] J.A. Harvey, P. Horava, and P. Kraus, "D-Sphalerons and The Topology of String Configuration Space," hep-th/0001143, JHEP 0002 (2000) 021.

[4] L. Houart and Y. Lozano, "Brane Descent Relations in M-theory," hepth/0001170, Phys. Lett. B479 (2000) 299; "S-Duality and Brane Descent Relations," hep-th/9911173, JHEP 0003 (2000) 031.

[5] P. Yi, "Membranes from five-branes and fundamental strings from Dp branes," hep-th/9901159, Nucl. Phys. B550, 214 (1999).

[6] P. Brax, G. Mandal, Y. Oz, "Supergravity Description of Non-BPS Branes," hep-th/0005242.

[7] A. Sen, private communication.

[8] O. Bergman, K. Hori and P. Yi, "Confinement on the brane," hepth/0002223, Nucl. Phys. B580, 289 (2000); 
G. Gibbons, K. Hori and P. Yi, "String fluid from unstable D-branes," hep-th/0009061.

[9] O. Bergman and M.R. Gaberdiel, "Non-BPS States in Heterotic-Type IIA Duality," hep-th/9901014, JHEP 9903 (1999) 013.

[10] M. Fabinger and P. Horava, "Casimir Effect Between World-Branes in Heterotic M-Theory," hep-th/0002073, Nucl. Phys. 580, 243 (2000).

[11] J.A. Harvey, P. Kraus, F. Larsen, "Tensionless Branes and Discrete Gauge Symmetry," hep-th/0008064, Phys. Rev. D63 (2001) 026002, and reference within to a private communication with A. Sen.

[12] E. Witten, "D-Branes and K Theory," hep-th/9810188, JHEP 019 (1998) 9812.

[13] P. Horava, "Type IIA D-Brane, K-Theory, and Matrix Theory," hepth/9812135, Adv.Theor.Math.Phys.2 (1999) 1373-1404.

[14] A. A. Gerasimov, S. L. Shatashvili, "On Exact Tachyon Potential in Open String Field Theory," hep-th/0009103, JHEP 0010 (2000) 034;

D. Kutasov, M. Marino, G. Moore, "Some Exact Results on Tachyon Condensation in String Field Theory," hep-th/0009148, JHEP 0010 (2000) 045. 